\title{
Tool Bits with Elastic Damping Inserted Elements Formed by Semi-Inserts with Different Rigidity
}

\author{
Sergey G. Novikov ${ }^{1, a}$, Vitaliy V. Malykhin $2, b$, Sergey A. Chevychelov², ${ }^{*}$ \\ and Fedor V. Novikov ${ }^{3, d}$ \\ ${ }^{1}$ Regional Open Social Institute, 85, Mayakovskogo ul. Kursk, 305009, Russia \\ 2 Southwest State University, 94, 50 Let Octyabrya ul., Kursk, 305040, Russia \\ ${ }^{3}$ Kharkiv National Economic University named after Semyon Kuznets, 9A, Nauli ul., Kharkiv, \\ 61166, Ukraine \\ anovikov.s.46@mail.ru, bmtio29@yandex.ru, ctschsa@yandex.ru, dfokusnik1@rambler
}

Keywords: Tool Bit, Holder, Vibration, Impact Load, Damping Semi-Inserts, Semi-Inserts Rigidity

\begin{abstract}
Vibration during turning is a consequence of premature tool wear and an increase in the roughness of parts. The designs of tool bits that increase their durability, reduce vibrations arising during the cutting process, and improve the quality of processing are of practical and scientific interest. Existing developments of vibration-damping bits with elastic damping inserts of constant rigidity, in which a holder or mandrel with a cut-off insert is installed, do not meet the requirements for the efficiency of vibration reduction due to the impossibility of the necessary vibration damping; the consumption of insert materials is high. Innovative designs of tool bits with inserts formed by semi-inserts with different rigidity are proposed, which allows improving the quality of processing by increasing the efficiency of vibration damping, reducing material consumption, because the length of the semi-inserts is less than half the length of the full insert. The tool bits can be used in the area of cutting materials, mechanical engineering and tool manufacturing.
\end{abstract}

\section{Introduction}

Reducing the vibration level during cutting is the most important problem, especially taking into account the increasing requirements for the quality of processing [1-4]. One of the ways to solve this problem is to improve the designs of cutting tools in order to increase the stability of the cutting process [5-10]. In particular, the designs of damping tool bits which make it possible to address the most important task of mechanical engineering to increase the efficiency of processing structural materials are being developed; this task involves achieving the highest productivity while ensuring a given level of quality of the surface layer of parts [11-15].

However, at present, the development of damping tool bits does not fully meet the imposed requirements. A significant drawback of most of the existing designs of tool bits is that they are rigidly attached to the machine [11-14], which does not allow achieving effective vibration reduction during processing. Many damping bits also have complex designs $[11,13,14]$, technologies of their manufacturing are very sophisticated, and normal operation of some machines is highly doubtful.

New designs of bits to increase their durability, reduce vibrations arising in the cutting process, and improve the quality of processing are of practical and scientific interest. 


\section{Experimental procedure}

Materials and methods for solving the problem. There is a damping tool bit containing a cut-off insert with a unit for its attachment in a holder which has a rabbet and an inserted element made of a material with a high damping property; the rabbet is made evenly along the perimeter of the end of the holder along the length from its end to the projection with the cut-off insert; the end of the holder with the rabbet is placed in a metal body made in the form of a rectangular parallelepiped with the same gaps along its base and walls and the gaps are filled with the material of the mentioned inserted element, and the holder is installed without the possibility of contact with the body [15]. The tool bit has the following drawbacks:

1. When turning a product, the perturbation cutting force causes vibrations of the tool bit, however, a material with a high damping property has constant rigidity for the solid inserted element, therefore, the pair of restoring forces arising in it, although creating a moment that makes the tool bit return to the position of static equilibrium and dampens vibrations, is not sufficient to minimize vibrations to the desired level, and does not allow achieving the required processing quality.

2. Excessive consumption of the material used for inserted element due to the fact that it fills the gaps along the entire length of the rabbet of the holder; the gaps between the surfaces of the holder with the rabbet and the inner cavity of the metal body have the form of rectangular parallelepipeds and are rather large.

The technical purpose of the use of the proposed damping tool bit is to improve the quality of processing and reduce the consumption of the material used for insert element.

Figure 1 shows a general view of the tool bit.
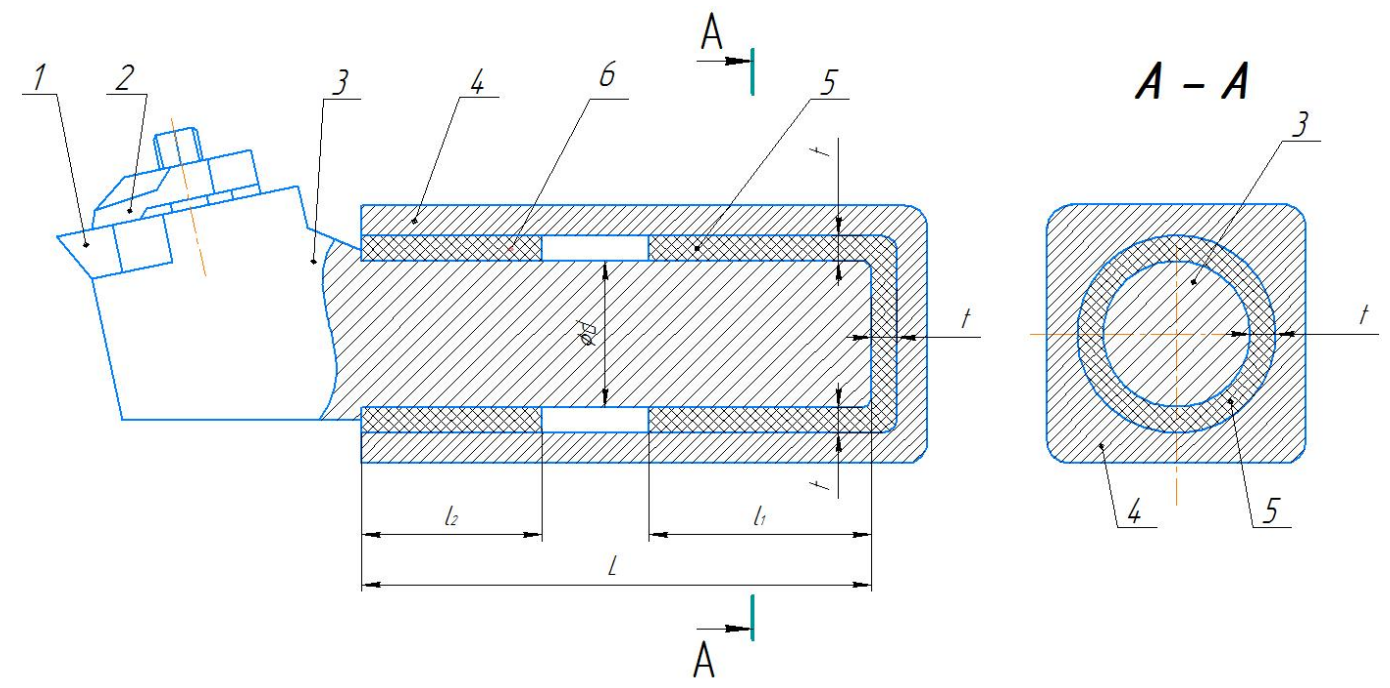

Fig. 1. A tool bit with damping semi-inserts of different rigidity:

1 -cut-off insert; 2 - insert fastening unit; 3 -holder; 4-body; 5, 6-damping semi-inserts.

The damping tool bit operates as follows. The required cutting conditions for structural material are specified. During workpiece processing, the perturbation cutting force causes the tool to vibrate. In the case of making the inserted element along the entire length $L$ of the cylindrical rabbet of holder 3 of diameter $d$ or consisting of semi-inserts 5, 6 of materials with high damping properties of the same rigidity $\left(\mathrm{j}_{1}=\mathrm{j}_{2}\right)$, a pair of restoring forces arise in the solid inserted element, and two identical pairs of restoring forces arise in semi-inserts 5, 6 creating moments that tend to 
return the bit to a static equilibrium position and damping vibrations, which are insufficient to minimize vibrations to the desired level.

In the proposed tool bit, the inserted element is formed of cylindrical semi-inserts 5,6 of materials with high damping properties of various rigidity $\left(\mathrm{j}_{1}>\mathrm{j}_{2}\right)$, and the lengths $\mathrm{l}_{1}, \mathrm{l}_{2}$ of semiinserts 5, 6 are less than half the length $\mathrm{L}$ of the rabbet $\left(1_{1}<\mathrm{L} / 2,1_{2}<\mathrm{L} / 2\right)$. Accordingly, in the semiinserts, there arise restoring parallel forces directed in opposite directions and not equal in modulus, while the moduli of forces in semi-insert 5 are more than the moduli of forces in semiinsertion 6. Therefore, in addition to the moment from a pair of forces equal in modulus to the forces in semi-inserts 5 and 6 , in semi-insert 5 there are still forces equal to the difference in force moduli in the semi-inserts and creating an additional restoring moment, which increases the efficiency of damping tool bit vibrations. (The additional moment is equal to the product of the difference between the moduli of the indicated forces by the arm). So, we get highly effective damping of longitudinal, transverse vibrations and impact loads arising in the cutting process; the reliability of this process increases in both turning and planing technological operations. Thus, by selecting different rigidity materials with the of high damping properties for semi-inserts 5,6 , it is possible to minimize tool bit vibrations to the required level and achieve the required processing quality.

The design allows:

1. To improve the quality of processing by increasing the efficiency of tool bit vibrations damping due to the use of the inserted element formed of two semi-inserts made of materials with high damping properties and having different rigidity; in this case, the regidity of the semi-insert located at the end of the holder at the bottom of the body base is higher than the rigidity of the semi-insert placed on opposite end of the holder with rabbet.

2. Reduce the consumption of the material used for inserted damping element, since it consists of two semi-inserts placed at a distance from each other; moreover, the length of both semi-inserts is less than half the length of the rabbet of the holder end; in addition, the insert fills the annular gap between the cylindrical rabbet of the holder and the cylindrical rabbet of the body, minimizing the consumption of materials used for semi-inserts.

This leads to the technical result of improving the quality of processing and reducing the consumption of the material used for inserts.

Results and discussion. The originality of the proposed damping tool bit is that in the rabbet made in the shape of mandrel 3, the lower end of mandrel 3 is placed with gaps $t$ uniform along the bottom and walls of the rabbet filled with an inserted element made of material with high damping properties, the inserted element is formed of two spaced apart from each other semiinserts made of materials with high damping properties and having different rigidity; the first of the semi-inserts - centre bearing 5 is placed at the end of mandrel 3 at the bottom of the rabbet, and the second semi-insert 6 is placed at the upper part of mandrel 3 placed in the rabbet; in this case, the length $h_{1}$ of centre bearing 5 and $h_{2}$ of the second semi-insert 6 is less than half the depth $\mathrm{h} / 2$ of the rabbet, and the rigidity of centre bearing 5 is higher than the rigidity of the second semiinsert 6, which makes it possible:

1. To improve the quality of processing by increasing the efficiency of damping vibrations of the cut-off insert rigidly mounted on the upper surface of the mandrel by forming an insert element from a centre bearing and a semi-insert, made of materials with high damping properties and having different rigidity, and the rigidity of the centre bearing is higher than the rigidity of the semi-insert. 
2. To simplify the design of the tool bit in such a way that the lower end of the mandrel is placed directly in the rabbet, made in the shape of the mandrel, with uniform gaps along the bottom and walls of the rabbet, which does not require additional structural elements to be placed.

3. Reduce the consumption of the inserted element material, since it consists of a centre bearing and a second semi-insert placed at the lower end of the mandrel at a distance from each other, and the length of the centre bearing and the semi-insert is less than half the depth of the rabbet; in addition, material consumption for manufacturing additional structural elements for placing the mandrel in the rabbet is not necessary.

\section{Summary}

Thus, the proposed damping tool bits make it possible to achieve a technical result to improve the quality of processing, simplify the design of tool bits and reduce material consumption for their manufacturing. The tool bits can be used in the field of materials cutting, mechanical engineering and tool production.

\section{References}

[1] Maslennikov A.V., Chevychelov S.A., Merzhoeva M.S., Gatiev M.S., Sidorova V.V. Influence of axial harmonic oscillations on chip formation when drilling holes in ductile metals. Russian Engineering Research. 2014. T. 34. № 11. C. 722-724. https://doi.org/10.3103/S1068798X14110136

[2] Grechukhin, A.N., Kuts, V.V., Razumov, M.S. Solving Problem of Curved Surface Approximation by Layers with Constant and Variable Sections During Forming by Additive Methods (2020) Lecture Notes in Mechanical Engineering, pp. 239-248. https://doi.org/10.1007/978-3-030-22041-9_28

[3] Kuts, V.V., Razumov, M.S., Sidorova, M.A. Development of the Internally Cutting Milling Cutter Model with Radial Advance for Treatment of Profiling Rolls (2017) Procedia Engineering, 206, pp. 1497-1502. https://doi.org/10.1016/j.proeng.2017.10.667

[4] Emelyanov S.G., Chevychelov S.A. Improving the quality of the processed surface by choosing a rational shaping scheme. Mechanical engineering and automation issues. 2004. No. 3. P. 86-88.

[5] Emelyanov S.G., Chevychelov S.A. Modelling the error of the profile formed by hyperbolic cutters. Bulletin of mechanical engineering. 2008. No. 1. P. 38-40.

[6] Maslennikov A.V., Chevychelov S.A., Golubev I.G. Rear angle of a helical bit in hole shaping with axial vibration. Russian Engineering Research. 2013. T. 33. № 5. C. 295-298. https://doi.org/10.3103/S1068798X13050092

[7] Razumov, M., Grechukhin, A., Maslennikov, A. Geometric Parameters of Cutting Tools that Can be Used for Forming Sided Surfaces with Variable Profile. Archives of Metallurgy and Materials, 2017, 62(1), стр. 33-40. https://doi.org/10.1515/amm-2017-0005

[8] Kuts, V.V., Gridin, D.S. Comprehensive study of the process of cutting screw grooves on the inner surface of a cylindrical steel sleeve with rotary cutting mandrels (2021) Chernye Metally, 2021 (2), pp. 63-68. https://doi.org/10.17580/chm.2021.02.11

[9] Kuts, V., Razumov, M., Kalutskikh, N. Modelling of cutting block cut surface at faceted surfaces machining using planetary gear set (2017) MATEC Web of Conferences, 129, статья № 01036. https://doi.org/10.1051/matecconf/201712901036 
[10] Grechishnikov, V.A., Kuts, V.V., Razumov, M.S., Romanov, V.B., Pivkin, P.M., Grechukhin, A.N., Yurasov, S.Y. Errors in shaping by a planetary mechanism (2017) Russian Engineering Research, 37 (9), pp. 824-826. https://doi.org/10.3103/S1068798X17090118 [11] Tool bit: RF Patent 2009768: MPK $^{5}$ B23B27/00/ V.A. Rogov; applicant patent holder Peoples' Friendship University of Russia. - No. 5014352/08; appl. 02.12.91; publ. 03.30.94.-3 p. [12] Tool bit: RF Patent 2280542: MPK $^{7}$ B23B27/00/ L.A. Vasin, N.N. Borodkin; applicant patent holder Tula State University. -25.04.2005; publ. 27.07.2006. 4 p.

[13] Tool bit: RF Patent 2287405: MPK B23B27/10/ G.M. Polezhaikin, N.A. Ladoshkin, A.G. Fomin L.A., I.G. Polezhaikin, T.G. Efanyeva; applicant - patent holder Mordovia State University named after N.P. Ogarev. No. 2005124 690/02; appl. 02.08.2005; publ. 20.11.2006. $3 \mathrm{p}$.

[14] Tool bit: RF Patent 2107588: MPK B23B27/16/ V.G. Voitenko, E.Yu. Tatarkin, A.A. Sitnikov; applicant-holder Altai State University. No96123454/02; appl. 15.12.96; publ. 27.03.98. 3p.

[15] Yatsun E.I., Malykhin V.V., Zubkova O.S. Novikov S.G. Instrumental support of machining processes with hard alloys and composites: monograph. Kursk: "Universitetskaya kniga". 2016. Pp.160-124 